Homology, Homotopy and Applications, vol. 17(1), 2015, pp.267-280

\title{
ALGEBRAIC PROOFS OF SOME FUNDAMENTAL THEOREMS IN ALGEBRAIC $K$-THEORY
}

\author{
TOM HARRIS
}

(communicated by Ulf Rehmann)

\begin{abstract}
We present new proofs of the additivity, resolution, and cofinality theorems for the algebraic $K$-theory of exact categories. These proofs are entirely algebraic, based on Grayson's presentation of higher algebraic $K$-groups via binary complexes.
\end{abstract}

\section{Introduction}

The beautiful and relatively young discipline of algebraic $K$-theory has seen tremendous development and far-reaching applications in many other mathematical disciplines over the last decades. This paper makes a contribution to a project (begun in Gra12] and Gra13]) reformulating its foundations.

The algebraic $K$-theory of an exact category was first described by Segal and Waldhausen, obtained by modifying Segal's construction of the $K$-theory of a symmetric monoidal category. Quillen's alternative $Q$-construction gives a very powerful tool for proving fundamental theorems in algebraic $K$-theory, which he exploited to prove the additivity, resolution, dévissage, and localisation theorems Qui73. Waldhausen's later work on the $S$.-construction, in particular his version of the additivity theorem, made simpler proofs of the theorems cited above and the cofinality theorem possible Sta89. Common in all of these approaches is the use of some non-trivial content from homotopy theory.

Grayson Gra12 recently gave the first presentation of the higher algebraic $K$ groups of an exact category by generators and relations; we take this presentation as our definition of $K_{n} \mathcal{N}$. The object of this paper is to present new completely algebraic proofs of the additivity, resolution, and cofinality theorems in higher algebraic $K$ theory of exact categories.

We assume throughout that the reader is familiar with exact categories. They are first systematically defined in Qui73; a very nice exposition is Büh10. In section 2 we review the necessary details of Grayson's presentation and present a new proof of the additivity theorem, in its form concerning so-called extension categories.

Theorem 1.1 (Additivity). Let $\mathcal{B}$ be an exact category, with exact subcategories $\mathcal{A}$ and $\mathcal{C}$ closed under extensions in $\mathcal{B}$. Let $\mathcal{E}(\mathcal{A}, \mathcal{B}, \mathcal{C})$ denote the associated extension category. Then $K_{n} \mathcal{E}(\mathcal{A}, \mathcal{B}, \mathcal{C}) \cong K_{n} \mathcal{A} \times K_{n} \mathcal{C}$, for every $n \geqslant 0$.

Received November 28, 2013, revised June 10, 2014; published on April 29, 2015.

2010 Mathematics Subject Classification: 19D99.

Key words and phrases: higher algebraic $K$-group, acyclic binary complex, additivity theorem, resolution theorem, cofinality theorem.

Article available at http://dx.doi.org/10.4310/HHA.2015.v17.n1.a13

Copyright (c) 2015, Tom Harris. Permission to copy for private use granted. 
Using Grayson's presentation, the proof of the additivity theorem is rather simple. In sections 3 and 4 we present more involved proofs of the resolution and cofinality theorems.

Theorem 1.2 (Resolution). Let $\mathcal{M}$ be an exact category and let $\mathcal{P}$ be a full, additive subcategory that is closed under extensions. Suppose also that:

1. If $0 \rightarrow M^{\prime} \rightarrow M \rightarrow M^{\prime \prime} \rightarrow 0$ is an exact sequence in $\mathcal{M}$ with $M$ and $M^{\prime \prime}$ in $\mathcal{P}$, then $M^{\prime}$ is in $\mathcal{P}$ as well.

2. Given $j: M \rightarrow P$ in $\mathcal{M}$ with $P$ in $\mathcal{P}$, there exists $j^{\prime}: P^{\prime} \rightarrow P$ and $f: P^{\prime} \rightarrow M$ in $\mathcal{M}$ with $P^{\prime}$ in $\mathcal{P}$ such that $j f=j^{\prime}$.

3. Every object of $\mathcal{M}$ has a finite resolution by objects of $\mathcal{P}$.

Then the inclusion functor $\mathcal{P} \hookrightarrow \mathcal{M}$ induces an isomorphism $K_{n}(\mathcal{P}) \cong K_{n}(\mathcal{M})$ for every $n \geqslant 0$.

Theorem 1.3 (Cofinality). Let $\mathcal{M}$ be a cofinal exact subcategory of an exact category $\mathcal{N}$. Then the inclusion functor $\mathcal{M} \hookrightarrow \mathcal{N}$ induces an injection $K_{0} \mathcal{M} \hookrightarrow K_{0} \mathcal{N}$ and isomorphisms $K_{n} \mathcal{M} \cong K_{n} \mathcal{N}$ for $n>0$.

Grayson defines the $n^{\text {th }}$ algebraic $K$-group of an exact category, denoted $K_{n} \mathcal{N}$, as a quotient group of the Grothendieck group of a certain related exact category $\left(B^{\mathrm{q}}\right)^{n} \mathcal{N}$, whose objects are so-called acyclic binary multicomplexes (see Definition2.5). Each of the theorems above establishes isomorphisms between the $K$-groups of exact categories. These theorems have well-known algebraic folk proofs for the Grothendieck group $K_{0}$, so the general schema for our proofs is then as follows. First we verify that the hypotheses on our exact categories of interest also hold for their associated categories of acyclic binary multicomplexes. Then we apply the algebraic $K_{0}$ proof to obtain an isomorphism between their Grothendieck groups. Finally we verify that the required isomorphism still holds when we pass to the quotients defining the higher algebraic $K$-groups.

The remaining theorems regarded as fundamental in the algebraic $K$-theory of exact categories are the dévissage and localisation theorems. These theorems concern abelian categories, say $\mathcal{A}$ and $\mathcal{B}$. While the associated categories $\left(B^{\mathrm{q}}\right)^{n} \mathcal{A}$ and $\left(B^{\mathrm{q}}\right)^{n} \mathcal{B}$ are still exact, they will no longer be abelian, so a strategy more sophisticated than the approach of this paper will be necessary to prove these theorems in the context of Grayson's new definition of the higher algebraic $K$-groups.

\section{Acknowledgments}

The author thanks Daniel Grayson for an essential insight into the proof of the resolution theorem, for the results of his paper [Gra12], which we draw upon extensively, and for his helpful comments on a late draft of this paper. Thanks are also due to the anonymous referee for suggesting a more natural induction argument for the proof of the cofinality theorem, as well as for spotting several unclarities and other small errors. Finally, the author is grateful to his supervisor Bernhard Köck for his careful readings of successive drafts of this work, for suggesting numerous improvements to its content, and for his encouragement and enthusiasm. 


\section{Grayson's binary complex algebraic $K$-theory}

In this section we recall the definitions and main result of Gra12. As a first application we give a simple new proof of the additivity theorem, as previously proven in Qui73, as well as McC93 and Gra11, and whose version for the $S$.-construction is commonly considered to be the fundamental theorem in the algebraic $K$-theory of spaces. We shall work throughout with exact categories in the sense of Quillen Qui73, that is, additive categories with a distinguished collection of short exact sequences that satisfies a certain set of axioms.

Definition 2.1. A bounded acyclic complex, or long exact sequence, in an exact category $\mathcal{N}$ is a bounded chain complex $N$ whose differentials factor through short exact sequences of $\mathcal{N}$. That is, the differentials $d_{k}: N_{k} \rightarrow N_{k-1}$ factor as $N_{k} \rightarrow Z_{k-1} \rightarrow$ $N_{k-1}$ such that each $0 \rightarrow Z_{k} \rightarrow N_{k} \rightarrow Z_{k-1} \rightarrow 0$ is a short exact sequence of $\mathcal{N}$.

In an abelian category the long exact sequences defined above agree with the usual long exact sequences. Care must be taken in the case of a general exact category, as the following example shows.

Example 2.2. Let $R$ be a ring with a finitely-generated, stably-free, non-free projective module $P$ (so $P \oplus R^{m} \cong R^{n}$ for some $m$ and $n$ ). We have short exact sequences of $R$-modules $0 \rightarrow P \stackrel{i}{\rightarrow} R^{n} \stackrel{p}{\rightarrow} R^{m} \rightarrow 0$ and $0 \rightarrow R^{m} \stackrel{j}{\rightarrow} R^{n} \stackrel{q}{\rightarrow} P \rightarrow 0$, where $i, j, p$, and $q$ are the obvious inclusions and projections. The sequence

$$
0 \longrightarrow R^{m} \stackrel{j}{\longrightarrow} R^{n} \stackrel{i q}{\longrightarrow} R^{n} \stackrel{p}{\longrightarrow} R^{n} \longrightarrow 0
$$

is a chain complex in the exact category of finitely-generated free modules, $\operatorname{Free}(R)$, and it is exact as a sequence of $R$ modules, but, in the sense of the definition above, it is not long exact in $\operatorname{Free}(R)$.

The category $C^{\mathrm{q}} \mathcal{N}$ of bounded acycli1 1 complexes in an exact category $\mathcal{N}$ is itself an exact category (Gra12,, (6); a sequence of chain maps $0 \rightarrow N^{\prime} \stackrel{\phi}{\rightarrow} N \stackrel{\psi}{\rightarrow} N^{\prime \prime} \rightarrow 0$ is said to be a short exact sequence if and only if $0 \rightarrow N_{k}^{\prime} \stackrel{\phi_{k}}{\rightarrow} N_{k} \stackrel{\psi_{k}}{\rightarrow} N_{k}^{\prime \prime} \rightarrow 0$ is short exact for all $k$. A word of warning here: a morphism of acyclic complexes $N \stackrel{\psi}{\rightarrow} N^{\prime \prime}$ such that every $N_{k} \stackrel{\psi_{k}}{\rightarrow} N_{k}^{\prime \prime}$ is an admissible epimorphism of $\mathcal{N}$ is not necessarily an admissible epimorphism in $C^{\mathrm{q}} \mathcal{N}$.

Example 2.3. Let $i, j, p$, and $q$ be the morphisms in Example 2.2, and note that $i q+$ $j p=1$. The diagram below has exact rows, and is in fact a morphism of $C^{\mathrm{q}} \mathbf{F r e e}(R)$.

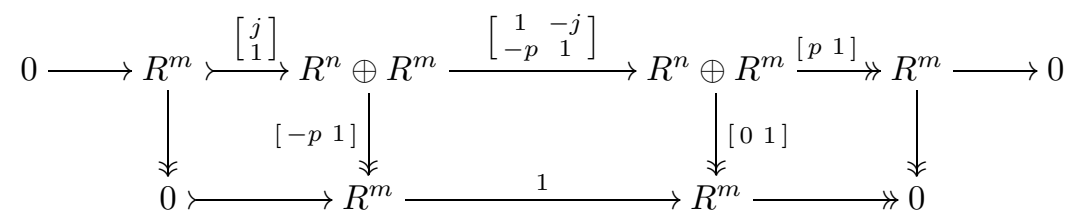

Each vertical arrow is an admissible epimorphism of $\operatorname{Free}(R)$, but the diagram is

\footnotetext{
${ }^{1}$ The q in $C^{\mathrm{q}} \mathcal{N}$ stands for "quasi-isomorphic to the zero complex." We will not make use of the notion of a quasi-isomorphism - the $\mathrm{q}$ is a reminder that we are dealing only with the acyclic complexes in $\mathcal{N}$.
} 
not an admissible epimorphism of $C^{\mathrm{q}} \mathbf{F r e e}(R)$-its kernel is the complex discussed in Example 2.2, which is not acyclic in $\operatorname{Free}(R)$.

Definition 2.4. A binary complex in $\mathcal{N}$ is a chain complex with two independent differentials. More precisely, a binary complex is a triple $(N, d, \tilde{d})$ such that $(N, d)$ and $(N, \tilde{d})$ are chain complexes in $\mathcal{N}$. We call a binary chain complex acyclic if each of the complexes $(N, d)$ and $(N, \tilde{d})$ is acyclic in $\mathcal{N}$. A morphism between binary complexes is a morphism between the underlying graded objects that commutes with both differentials. A short exact sequence is a composable pair of such morphisms that is short exact in every degree.

Since $C^{\mathrm{q}} \mathcal{N}$ is an exact category, the reader may easily check that the category $B^{\mathrm{q}} \mathcal{N}$ of bounded acyclic binary complexes in $\mathcal{N}$ is also an exact category. There is a diagonal functor $\Delta: C^{\mathrm{q}} \mathcal{N} \rightarrow B^{\mathrm{q}} \mathcal{N}$, sending $(N, d)$ to $(N, d, d)$. A binary complex that is in the image of $\Delta$ is also called diagonal. The diagonal functor is split by the top and bottom functors $\top, \perp: B^{\mathrm{q}} \mathcal{N} \rightarrow C^{\mathrm{q}} \mathcal{N}$; it is clear that $\Delta, \top$, and $\perp$ are all exact.

Taking the category of acyclic binary complexes behaves well with respect to subcategories closed under extensions. If $\mathcal{M}$ is a full subcategory closed under extension in $\mathcal{N}$ (later just called an exact subcategory), then $B^{\mathrm{q}} \mathcal{M}$ is a subcategory closed under extensions in $B^{\mathrm{q}} \mathcal{N}$. It is important here that the binary complexes in $B^{\mathrm{q}} \mathcal{N}$ are bounded. Starting at the final non-zero term, one argues by induction that the objects that the extension factors through are actually in $\mathcal{M}$, using the hypothesis on $\mathcal{M}$ and $\mathcal{N}$, and the $3 \times 3$ Lemma ([B̈̈h10], Corollary 3.6).

Since $B^{\mathrm{q}} \mathcal{N}$ is an exact category, we can iteratively define for each $n \geqslant 0$ an exact category $\left(B^{\mathrm{q}}\right)^{n} \mathcal{N}=B^{\mathrm{q}} B^{\mathrm{q}} \ldots B^{\mathrm{q}} \mathcal{N}$. The objects of this category are bounded acyclic binary complexes of bounded acyclic binary complexes ... of objects of $\mathcal{N}$. Happily, this may be neatly unwrapped: the following is an equivalent definition of $\left(B^{\mathrm{q}}\right)^{n} \mathcal{N}$.

Definition 2.5. The exact category $B^{\mathrm{q}} \mathcal{N}$ of bounded acyclic binary multicomplexes of dimension $n$ in $\mathcal{N}$ is defined as follows. A bounded acyclic binary multicomplex of dimension $n$ is a $\mathbb{Z}^{n}$-graded collection of objects of $\mathcal{N}$, only finitely many of which are non-zero, together with a pair of acyclic differentials $d^{i}$ and $\tilde{d}^{i}$ in each direction $1 \leqslant i \leqslant n$ such that, for $i \neq j$,

$$
\begin{aligned}
& d^{i} d^{j}=d^{j} d^{i} \\
& d^{i} \tilde{d}^{j}=\tilde{d}^{j} d^{i} \\
& \tilde{d}^{i} d^{j}=d^{j} \tilde{d}^{i} \\
& \tilde{d}^{i} \tilde{d}^{j}=\tilde{d}^{j} \tilde{d}^{i} .
\end{aligned}
$$

In other words, any pair of differentials in different directions commutes. A morphism $\phi: N \rightarrow N^{\prime}$ between such binary multicomplexes is a $\mathbb{Z}^{n}$-graded collection of morphisms of $\mathcal{N}$ that commutes with all of the differentials of $N$ and $N^{\prime}$. A short exact sequence in $\left(B^{\mathrm{q}}\right)^{n} \mathcal{N}$ is a composable pair of such morphisms that is short exact in every degree.

In addition to $\left(B^{\mathrm{q}}\right)^{n} \mathcal{N}$, for $n \geqslant 1$ we have exact categories $\left(B^{\mathrm{q}}\right)^{i-1} C^{\mathrm{q}}\left(B^{\mathrm{q}}\right)^{n-i} \mathcal{N}$ for $i=1, \ldots n$, each of which is equivalent to $C^{\mathrm{q}}\left(B^{\mathrm{q}}\right)^{n-1} \mathcal{N}$, the category of acyclic complexes of objects of $\left(B^{\mathrm{q}}\right)^{n-1} \mathcal{N}$. For each $i$ with $1 \leqslant i \leqslant n$ there is a diagonal functor $\Delta_{i}:\left(B^{\mathrm{q}}\right)^{i-1} C^{\mathrm{q}}\left(B^{\mathrm{q}}\right)^{n-i} \mathcal{N} \rightarrow\left(B^{\mathrm{q}}\right)^{n} \mathcal{N}$ that consists of "doubling up" the differential 
of the (non-binary) acyclic complex and regarding it as providing both differentials in the direction $i$ in the resulting acyclic binary multicomplex. Any object of $\left(B^{\mathrm{q}}\right)^{n} \mathcal{N}$ that is in the image of one of these $\Delta_{i}$ is called diagonal. The diagonal binary multicomplexes are those that have $d^{i}=\tilde{d}^{i}$ for at least one $i$.

We can now formulate Grayson's presentation of the algebraic $K$-theory groups of $\mathcal{N}$, which we shall take to be their definition for the remainder of this paper.

Theorem / Definition 2.6 (Gra12, Corollary 7.4). For $\mathcal{N}$ an exact category and $n \geqslant 0$, the abelian group $K_{n} \mathcal{N}$ is presented as follows. There is one generator for each bounded acyclic binary multicomplex of dimension $n$, and there are relations $\left[N^{\prime}\right]+$ $\left[N^{\prime \prime}\right]=[N]$ if there is a short exact sequence $0 \rightarrow N^{\prime} \rightarrow N \rightarrow N^{\prime \prime} \rightarrow 0$ in $\left(B^{\mathrm{q}}\right)^{n} \mathcal{N}$, and $[T]=0$ if $T$ is a diagonal acyclic binary multicomplex.

Observe that if we disregard the second relation that diagonal binary multicomplexes vanish, then we obtain the Grothendieck group $K_{0}\left(B^{\mathrm{q}}\right)^{n} \mathcal{N}$. Another way to say this is that $K_{n} \mathcal{N}$ is a quotient group of the Grothendieck group of the exact category $\left(B^{\mathrm{q}}\right)^{n} \mathcal{N}$. Denote by $T_{\mathcal{N}}^{n}$ the subgroup of $K_{0}\left(B^{\mathrm{q}}\right)^{n} \mathcal{N}$ generated by the classes of the diagonal binary multicomplexes in $\left(B^{\mathrm{q}}\right)^{n} \mathcal{N}$. Then we may write $K_{n} \mathcal{N} \cong\left(K_{0}\left(B^{\mathrm{q}}\right)^{n} \mathcal{N}\right) / T_{\mathcal{N}}^{n}$.

Using the inductive viewpoint explained before Definition 2.5, we also obtain the following description of $K_{n} \mathcal{N}$.

Lemma 2.7. For each $n \geqslant 1$ we have a split short exact sequence:

$$
0 \longrightarrow K_{n-1} C^{\mathrm{q}} \mathcal{N} \stackrel{\Delta}{\longrightarrow} K_{n-1} B^{\mathrm{q}} \mathcal{N} \longrightarrow K_{n} \mathcal{N} \longrightarrow 0,
$$

which is functorial in $\mathcal{N}$.

Proof. This is proven already in Gra12, but a direct proof from Theorem / Definition [2.6] is instructive, so we include it here. We have defined $K_{n} \mathcal{N}$ to be $K_{0}\left(B^{\mathrm{q}}\right)^{n} \mathcal{N}$ modulo the sum of the images of the various diagonal maps

$$
\Delta_{i}: K_{0}\left(B^{\mathrm{q}}\right)^{i-1} C^{\mathrm{q}}\left(B^{\mathrm{q}}\right)^{n-i} \mathcal{N} \longrightarrow K_{0}\left(B^{\mathrm{q}}\right)^{n} \mathcal{N} \quad(i=1, \ldots, n) .
$$

Similarly, $K_{n-1} B^{\mathrm{q}} \mathcal{N}$ is defined as the factor group of $K_{0}\left(B^{\mathrm{q}}\right)^{n-1} B^{\mathrm{q}}=K_{0}\left(B^{\mathrm{q}}\right)^{n} \mathcal{N}$ modulo the sum of the images of $\Delta_{i}$ for $i=1, \ldots, n-1$. Therefore, the canonical epimorphism $K_{0}\left(B^{\mathrm{q}}\right)^{n} \mathcal{N}$ naturally factorises as

$$
K_{0}\left(B^{\mathrm{q}}\right)^{n} \mathcal{N} \longrightarrow K_{n-1} B^{\mathrm{q}} \mathcal{N} \longrightarrow K_{n} \mathcal{N}
$$

and $K_{n} \mathcal{N}$ is the factor group of $K_{n-1} B^{\mathrm{q}} \mathcal{N}$ modulo the image of the composition

$$
K_{0}\left(B^{\mathrm{q}}\right)^{n-1} C^{\mathrm{q}} \mathcal{N} \stackrel{\Delta_{n}}{\longrightarrow} K_{0}\left(B^{\mathrm{q}}\right)^{n} \mathcal{N} \longrightarrow K_{n-1} B^{\mathrm{q}} \mathcal{N} .
$$

Furthermore, the epimorphism $K_{0}\left(B^{\mathrm{q}}\right)^{n-1} \rightarrow K_{n-1}$ is a natural transformation of functors from the category of exact categories to abelian groups. Summarising, we obtain the following commutative diagram, whose bottom row is exact:

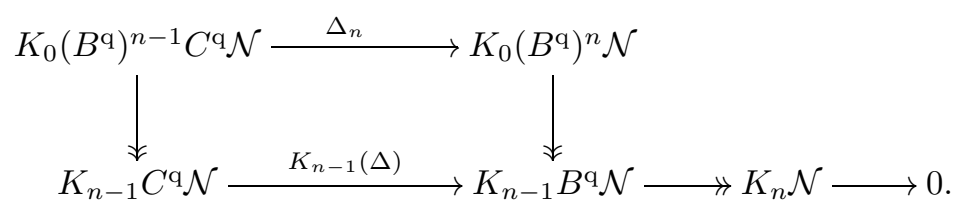


The top and bottom functors $\left(\top\right.$ and $\perp$ ) each split $\Delta$, so $K_{n-1}(\Delta)$ is split by either of $K_{n-1}(\top)$ or $K_{n-1}(\perp)$, so the bottom row of the diagram is our required split short exact sequence.

We now present a new, elementary proof of the additivity theorem. Let $\mathcal{A}$ and $\mathcal{C}$ be exact subcategories of an exact category $\mathcal{B}$.

Definition 2.8. The extension category $\mathcal{E}(\mathcal{A}, \mathcal{B}, \mathcal{C})$ is the exact category whose objects are short exact sequences $0 \rightarrow A \rightarrow B \rightarrow C \rightarrow 0$ in $\mathcal{B}$, with $A$ in $\mathcal{A}$ and $C$ in $\mathcal{C}$, and whose morphisms are commuting rectangles.

Theorem 2.9 (Additivity). $K_{n} \mathcal{E}(\mathcal{A}, \mathcal{B}, \mathcal{C}) \cong K_{n} \mathcal{A} \times K_{n} \mathcal{C}$, for every $n \geqslant 0$.

Proof. The exact functors

$$
\begin{aligned}
\mathcal{E}(\mathcal{A}, \mathcal{B}, \mathcal{C}) & \longrightarrow \mathcal{A} \times \mathcal{C} \\
(0 \rightarrow A \rightarrow B \rightarrow C \rightarrow 0) \longmapsto & \longmapsto(A, C)
\end{aligned}
$$

and

$$
\begin{aligned}
& \mathcal{A} \times \mathcal{C} \longrightarrow \mathcal{E}(\mathcal{A}, \mathcal{B}, \mathcal{C}) \\
& (A, C) \longmapsto(0 \rightarrow A \rightarrow A \oplus C \rightarrow C \rightarrow 0)
\end{aligned}
$$

induce mutually inverse isomorphisms between $K_{0} \mathcal{E}(\mathcal{A}, \mathcal{B}, \mathcal{C})$ and $K_{0} \mathcal{A} \times K_{0} \mathcal{C}$ (see, e.g., Wei13, II.9.3). Fix an $n>0$. The categories $\left(B^{\mathrm{q}}\right)^{n} \mathcal{A}$ and $\left(B^{\mathrm{q}}\right)^{n} \mathcal{C}$ are exact subcategories of $\left(B^{\mathrm{q}}\right)^{n} \mathcal{B}$, so we can define the extension category

$$
\mathcal{E}^{n}(\mathcal{A}, \mathcal{B}, \mathcal{C}):=\mathcal{E}\left(\left(B^{\mathrm{q}}\right)^{n} \mathcal{A},\left(B^{\mathrm{q}}\right)^{n} \mathcal{B},\left(B^{\mathrm{q}}\right)^{n} \mathcal{C}\right) .
$$

From the above, the induced map

$$
K_{0} \mathcal{E}^{n}(\mathcal{A}, \mathcal{B}, \mathcal{C}) \rightarrow K_{0}\left(B^{\mathrm{q}}\right)^{n} \mathcal{A} \times K_{0}\left(B^{\mathrm{q}}\right)^{n} \mathcal{C}
$$

is an isomorphism. But a short exact sequence of binary multicomplexes is exactly the same thing as a binary multicomplex of short exact sequences, so the categories $\mathcal{E}^{n}(\mathcal{A}, \mathcal{B}, \mathcal{C})$ and $\left(B^{\mathrm{q}}\right)^{n} \mathcal{E}(\mathcal{A}, \mathcal{B}, \mathcal{C})$ are isomorphic, so we therefore have an isomorphism

$$
K_{0}\left(B^{\mathrm{q}}\right)^{n} \mathcal{E}(\mathcal{A}, \mathcal{B}, \mathcal{C}) \cong K_{0}\left(B^{\mathrm{q}}\right)^{n} \mathcal{A} \times K_{0}\left(B^{\mathrm{q}}\right)^{n} \mathcal{C} .
$$

Identifying the categories $\mathcal{E}^{n}(\mathcal{A}, \mathcal{B}, \mathcal{C})$ and $\left(B^{\mathrm{q}}\right)^{n} \mathcal{E}(\mathcal{A}, \mathcal{B}, \mathcal{C})$, a binary multicomplex in $\left(B^{\mathrm{q}}\right)^{n} \mathcal{E}(\mathcal{A}, \mathcal{B}, \mathcal{C})$ is diagonal in direction $i$ if and only if its constituent binary multicomplexes in $\left(B^{\mathrm{q}}\right)^{n} \mathcal{A},\left(B^{\mathrm{q}}\right)^{n} \mathcal{B}$, and $\left(B^{\mathrm{q}}\right)^{n} \mathcal{C}$ are also diagonal in direction $i$. Similarly, if $A$ in $\left(B^{\mathrm{q}}\right)^{n} \mathcal{A}$ and $C$ in $\left(B^{\mathrm{q}}\right)^{n} \mathcal{C}$ are diagonal, then the binary multicomplexes corresponding to $(0 \rightarrow A \rightarrow A \rightarrow 0 \rightarrow 0)$ and $(0 \rightarrow 0 \rightarrow C \rightarrow C \rightarrow 0)$ are diagonal as well, so the isomorphism $K_{0}\left(B^{\mathrm{q}}\right)^{n} \mathcal{E}(\mathcal{A}, \mathcal{B}, \mathcal{C}) \cong K_{0}\left(B^{\mathrm{q}}\right)^{n} \mathcal{A} \times K_{0}\left(B^{\mathrm{q}}\right)^{n} \mathcal{C}$ restricts to an isomorphism $T_{\mathcal{E}(\mathcal{A}, \mathcal{B}, \mathcal{C})}^{n} \cong T_{\mathcal{A}}^{n} \times T_{\mathcal{B}}^{n}$. Passing to the quotients yields the result.

\section{The resolution theorem}

The resolution theorem relates the $K$-theory of an exact category, to that of a larger exact category, all of whose objects have a finite resolution by objects of the first category. Its most well-known application states that the $K$-theory of a regular 
ring is isomorphic to its $G$-theory (the $K$-theory of the exact category of all finitelygenerated $R$-modules). As in the proof of the additivity theorem, we adapt a simple proof for $K_{0}$ to work for all $K_{n}$. The main difficulty in this proof is verifying that the hypotheses of the theorem pass to exact categories of acyclic binary multicomplexes.

The general resolution theorem for exact categories (Qui73, $\S 4$ Corollary 2) is a formal consequence of the following theorem, which is Theorem 3 of Qui73.

Theorem 3.1. Let $\mathcal{P}$ be a full, additive subcategory of an exact category $\mathcal{M}$ that is closed under extensions and satisfies:

1. For any exact sequence $0 \rightarrow P^{\prime} \rightarrow P \rightarrow M \rightarrow 0$ in $\mathcal{M}$, if $P$ is in $\mathcal{P}$, then $P^{\prime}$ is in $\mathcal{P}$.

2. For any $M$ in $\mathcal{M}$ there exists a $P$ in $\mathcal{P}$ and an admissible epimorphism $P \rightarrow M$.

Then the inclusion functor $\mathcal{P} \hookrightarrow \mathcal{M}$ induces an isomorphism $K_{n} \mathcal{P} \cong K_{n} \mathcal{M}$ for all $n \geqslant 0$.

Proof. For $K_{0}$ the inverse to the induced homomorphism $K_{0} \mathcal{P} \rightarrow K_{0} \mathcal{M}$ is given by the map

$$
\begin{aligned}
\phi: K_{0} \mathcal{M} & \longmapsto K_{0} \mathcal{P} \\
{[M] } & \longmapsto[P]-\left[P^{\prime}\right]
\end{aligned}
$$

where $0 \rightarrow P^{\prime} \rightarrow P \rightarrow M \rightarrow 0$ is a short exact sequence of $\mathcal{M}$. The proof of Theorem 3.1 for $n=0$ is the simple exercise of checking that $\phi$ is well-defined. We noted earlier that if $\mathcal{P}$ is closed under extensions in $\mathcal{M}$, then $\left(B^{\mathrm{q}}\right)^{n} \mathcal{P}$ is closed under extensions in $\left(B^{\mathrm{q}}\right)^{n} \mathcal{M}$ for each $n$, and by the same reasoning, one easily sees that if $\mathcal{P}$ and $\mathcal{M}$ satisfy hypothesis (1) of the theorem, then so do $\left(B^{\mathrm{q}}\right)^{n} \mathcal{P}$ and $\left(B^{\mathrm{q}}\right)^{n} \mathcal{M}$ for each $n$. The following proposition is about hypothesis (2).

Proposition 3.2. Let $\mathcal{P}$ and $\mathcal{M}$ satisfy the hypotheses of [3.1. For every object $M$ of $\left(B^{\mathrm{q}}\right)^{n} \mathcal{M}$ there exists a short exact sequence $0 \rightarrow P^{\prime} \rightarrow P \rightarrow M \rightarrow 0$ of $\left(B^{\mathrm{q}}\right)^{n} \mathcal{M}$ with $P^{\prime}$ and $P$ in $\left(B^{\mathrm{q}}\right)^{n} \mathcal{P}$. Furthermore, if $M$ is a diagonal binary multicomplex, then we may choose $P$ and $P^{\prime}$ to be diagonal as well.

We shall prove Proposition 3.2 shortly. We now continue with the proof of Theorem 3.1. Together with the known isomorphism for $K_{0}$, the first part of the proposition implies that the induced map $K_{0}\left(B^{\mathrm{q}}\right)^{n} \mathcal{P} \rightarrow K_{0}\left(B^{\mathrm{q}}\right)^{n} \mathcal{M}$ is an isomorphism for each $n$. Clearly this isomorphism sends elements of $T_{\mathcal{P}}^{n}$ to elements of $T_{\mathcal{M}}^{n}$. Since the value of $\phi$ is independent of the choice of resolution, the second part of the proposition implies that $\phi$ maps elements of $T_{\mathcal{M}}^{n}$ to elements of $T_{\mathcal{P}}^{n}$. The isomorphism therefore descends to an isomorphism $K_{n} \mathcal{P} \rightarrow K_{n} \mathcal{M}$.

It remains then to prove Proposition 3.2. so for the rest of this section we assume the hypotheses of Theorem 3.1. The idea of the proof is to construct, for each $M$ in $\left(B^{\mathrm{q}}\right)^{n} \mathcal{M}$, a morphism of acyclic binary chain complexes $P \rightarrow M$ that is an admissible epimorphism in every degree, i.e., $P_{j} \rightarrow M_{j}$ is admissible for each $j$. By the assumption on $\mathcal{P}$ and $\mathcal{M}$ each of these admissible epimorphisms is part of a short exact sequence $0 \rightarrow P_{j}^{\prime} \rightarrow P_{j} \rightarrow M_{j} \rightarrow 0$ with the $P_{j}$ in $\mathcal{P}$. The $P_{j}^{\prime}$ form a binary complex with the induced maps, and we show that this binary complex is in fact acyclic. The result will then follow from an induction on the dimension. We shall rely on the following facts. 
Lemma 3.3. Let $f_{i}: Q_{i} \rightarrow N, i=1, \ldots, m$ be a family of morphisms in an exact category, at least one of which is an admissible epimorphism. Then the induced morphism

$$
\left[\begin{array}{lll}
f_{1} & \ldots & f_{m}
\end{array}\right]: \bigoplus_{i=1}^{m} Q_{i} \rightarrow N
$$

is an admissible epimorphism as well.

Proof. The general case follows from the case $m=2$. In this case, the morphism $\left[\begin{array}{ll}f_{1} & f_{2}\end{array}\right]$ factors as the composition

$$
Q_{1} \oplus Q_{2} \stackrel{\left[\begin{array}{ll}
f_{1} & 0 \\
0 & 1
\end{array}\right]}{\longrightarrow} N \oplus Q_{2} \stackrel{\left[\begin{array}{ll}
1 & f_{2} \\
0 & 1
\end{array}\right]}{\longrightarrow} N \oplus Q_{2} \stackrel{\left[\begin{array}{ll}
1 & 0
\end{array}\right]}{\longrightarrow} N
$$

all of which are admissible epimorphisms.

Lemma 3.4. Let $0 \rightarrow A \rightarrow B \rightarrow C \rightarrow 0$ be a short exact sequence of bounded chain complexes in an exact category $\mathcal{N}$ such that $B$ and $C$ are acyclic. Suppose that the differentials of $B$ and $C$ factor through short exact sequences $0 \rightarrow Y_{j} \rightarrow B_{j} \rightarrow Y_{j-1} \rightarrow$ 0 and $0 \rightarrow Z_{j} \rightarrow C_{j} \rightarrow Z_{j-1} \rightarrow 0$ of $\mathcal{N}$. Then $A$ is acyclic if and only if each of the induced morphisms $Y_{j} \rightarrow Z_{j}$ is an admissible epimorphism in $\mathcal{N}$. In this case the differentials of $A$ factor through the kernels $X_{j}$ of $Y_{j} \rightarrow Z_{j}$.

Proof. Without loss of generality, assume that $A, B$, and $C$ are all 0 in negative degrees, so that $Y_{0}=B_{0}$ and $Z_{0}=C_{0}$. Define $X_{0}$ to be $A_{0}$, the kernel of $Y_{0} \rightarrow Z_{0}$. Since $Y_{1} \rightarrow Z_{1}$ is an admissible epimorphism of $\mathcal{N}$, it has a kernel, which we shall denote by $X_{1}$. There are induced maps of kernels $X_{1} \rightarrow A_{1} \rightarrow X_{0}$, and since $0 \rightarrow Y_{1} \rightarrow$ $B_{1} \rightarrow Y_{0} \rightarrow 0$ and $0 \rightarrow Z_{1} \rightarrow C_{1} \rightarrow Z_{0} \rightarrow 0$ are short exact, then so is $0 \rightarrow X_{1} \rightarrow$ $A_{1} \rightarrow X_{0} \rightarrow 0$ by the $3 \times 3$ Lemma for exact categories. Defining $X_{j}$ to be the kernel of the admissible epimorphism $Y_{j} \rightarrow Z_{j}$ for each $j$, this argument is repeated to show that $0 \rightarrow X_{j} \rightarrow A_{j} \rightarrow X_{j-1} \rightarrow 0$ is short exact for every $j$. Uniqueness of induced maps between kernels shows that the differentials of $A$ factor through these short exact sequences, so $A$ is acyclic.

We begin resolving binary complexes in the less involved case, in which we assume $M$ to be diagonal.

Lemma 3.5. Given a diagonal bounded acyclic binary complex $M$ in $B^{\mathrm{q}} \mathcal{M}$, there exists a short exact sequence $0 \rightarrow P^{\prime} \rightarrow P \rightarrow M \rightarrow 0$ where $P^{\prime}$ and $P$ are diagonal objects of $B^{\mathrm{q}} \mathcal{P}$.

Proof. We may consider $M$ as an object of $C^{\mathrm{q}} \mathcal{N}$, as $\Delta: C^{\mathrm{q}} \mathcal{N} \rightarrow B^{\mathrm{q}} \mathcal{N}$ is a full embedding for any exact category $\mathcal{N}$. Represent $M$ in $C^{\mathrm{q}} \mathcal{N}$ as below. Without loss of generality we assume that $M$ ends at place 0 .

$$
0 \longrightarrow M_{n} \stackrel{d}{\longrightarrow} \cdots \stackrel{d}{\longrightarrow} M_{k} \stackrel{d}{\longrightarrow} M_{k-1} \stackrel{d}{\longrightarrow} \cdots \stackrel{d}{\longrightarrow} M_{0} \longrightarrow 0 .
$$

Since $\mathcal{P}$ and $\mathcal{M}$ satisfy the hypotheses of Theorem 3.1, there exists an object $Q_{k}$ of $\mathcal{P}$ and an admissible epimorphism $\epsilon_{k}: Q_{k} \rightarrow M_{k}$ in $\mathcal{M}$ for each $0 \leqslant k \leqslant n$. The diagram 
below is a morphism in $B^{\mathrm{q}} \mathcal{M}$ with target $M$, and its upper row, the source of the morphism, is an object of $C^{\mathrm{q}} \mathcal{P}$.

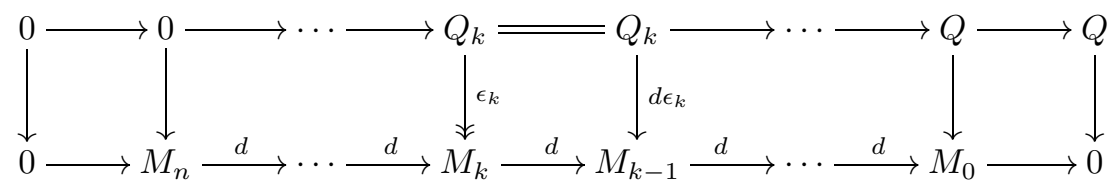

We denote the top row by $P^{k}$ and the morphism of complexes by $\zeta^{k}: P^{k} \rightarrow M$. We do this for each $k \in\{0, \ldots, n\}$ and form the sum

$$
\zeta:=\left[\begin{array}{lll}
\zeta^{n} & \ldots & \zeta^{0}
\end{array}\right]: \bigoplus P^{k} \rightarrow M
$$

Call the direct sum $P$. Each of the complexes $P^{k}$ is acyclic, so $\mathcal{P}$ is as well. Consider the morphism $\zeta_{j}: P_{j} \rightarrow M_{j}$, the part of $\zeta$ from the $j^{\text {th }}$ term of $P_{j}$ to the $j^{\text {th }}$ term of $M$. By construction and Lemma 3.3, it is an admissible epimorphism of $\mathcal{M}$. We now have a morphism $\zeta: P \rightarrow M$ that is an admissible epimorphism in every degree. The kernels of these admissible epimorphisms are all in $\mathcal{P}$ by the hypotheses on $\mathcal{P}$ and $\mathcal{M}$. These kernels form a (as we have seen in Example 2.3, not a priori acyclic) bounded chain complex $P^{\prime}$ in $\mathcal{P}$ under the induced maps between them.

It remains to show that this chain complex is acyclic (i.e., that $P^{\prime}$ is in $B^{\mathrm{q}} \mathcal{P}$ ), for then $0 \rightarrow P^{\prime} \rightarrow P \rightarrow M \rightarrow 0$ will be a short exact sequence of acyclic complexes. Suppose that the differential on $M$ factors through objects $Z_{j}$ of $\mathcal{M}$, and that $P$ factors through objects $Y_{j}$ of $\mathcal{P}$. By Lemma 3.4, to show that $P^{\prime}$ is acyclic in $\mathcal{M}$ it is enough to show that each $Y_{j} \rightarrow Z_{j}$ is an admissible epimorphism of $\mathcal{M}$. Since each $P^{k}$ is concentrated in degrees $k$ and $k-1$, we have $Y_{j}=Q_{j}$ and the induced map $Q_{j} \rightarrow Z_{j}$ is the composition $Q_{j} \rightarrow M_{j} \rightarrow Z_{j}$, which is an admissible epimorphism of $\mathcal{M}$, so $P^{\prime}$ is acyclic in $\mathcal{M}$ and its differentials factor through the kernels of the admissible epimorphisms $Q_{j} \rightarrow Z_{j}$ (call them $X_{j}$ ). But each $Q_{j}$ is an object of $\mathcal{P}$, and so each $X_{j}$ is an object of $\mathcal{P}$ as well, by the hypotheses on $\mathcal{P}$ and $\mathcal{M}$, so $P^{\prime}$ is acyclic in $\mathcal{P}$.

Finally we consider $P^{\prime}, P$, and $M$ as diagonal binary complexes (by applying $\Delta$ ). Then $0 \rightarrow P^{\prime} \rightarrow P \rightarrow M \rightarrow 0$ is the required short exact sequence of acyclic diagonal binary complexes.

A little more work is required if the binary complex $M$ is not diagonal. The idea in this case is due to Grayson, and relies on the acyclicity of the chain complexes

$$
0 \longrightarrow Q \stackrel{\left[\begin{array}{l}
1 \\
0
\end{array}\right]}{\longrightarrow} Q \oplus Q \stackrel{\left[\begin{array}{ll}
0 & 1 \\
0 & 0
\end{array}\right]}{\longrightarrow} Q \oplus Q \stackrel{\left[\begin{array}{ll}
0 & 1 \\
0 & 0
\end{array}\right]}{\longrightarrow} \cdots \stackrel{\left[\begin{array}{ll}
0 & 1 \\
0 & 0
\end{array}\right]}{\longrightarrow} Q \oplus Q \stackrel{\left[\begin{array}{ll}
0 & 1
\end{array}\right]}{\longrightarrow} Q \longrightarrow 0
$$

and

$$
0 \longrightarrow Q \stackrel{\left[\begin{array}{l}
0 \\
1
\end{array}\right]}{\longrightarrow} Q \oplus Q \stackrel{\left[\begin{array}{ll}
0 & 0 \\
1 & 0
\end{array}\right]}{\longrightarrow} Q \oplus Q \stackrel{\left[\begin{array}{ll}
0 & 0 \\
1 & 0
\end{array}\right]}{\longrightarrow} \cdots \stackrel{\left[\begin{array}{ll}
0 & 0 \\
1 & 0
\end{array}\right]}{\longrightarrow} Q \oplus Q \stackrel{\left[\begin{array}{ll}
1 & 0
\end{array}\right]}{\longrightarrow} Q \longrightarrow 0
$$

of arbitrary length, where $Q$ is an object of any exact category.

Lemma 3.6. Given an arbitrary bounded acyclic binary complex $M$ in $B^{\mathrm{q}} \mathcal{M}$ there exists a short exact sequence $0 \rightarrow P^{\prime} \rightarrow P \rightarrow M \rightarrow 0$, where $P^{\prime}$ and $P$ are objects of $B^{\mathrm{q}} \mathcal{P}$. 
Proof. Let $M$ denote the element of $B^{q} \mathcal{M}$ given by the binary complex below:

$$
0 \Longrightarrow M_{n} \underset{d^{\prime}}{\stackrel{d}{\rightrightarrows}} \cdots \underset{d^{\prime}}{\stackrel{d}{\rightrightarrows}} M_{k} \underset{d^{\prime}}{\stackrel{d}{\rightrightarrows}} M_{k-1} \underset{d^{\prime}}{\stackrel{d}{\rightrightarrows}} \cdots \underset{d^{\prime}}{\stackrel{d}{\rightrightarrows}} M_{0} \Longrightarrow 0
$$

and as before, for $0 \leqslant k \leqslant n$, let $\epsilon_{k}: Q_{k} \rightarrow M_{k}$ be admissible epimorphisms in $\mathcal{M}$ with $Q_{k}$ in $\mathcal{P}$. For $0 \leqslant k \leqslant n$ and $1 \leqslant l \leqslant k$, inductively define two collections of morphisms $\delta_{k, l}, \delta_{k, l}^{\prime}: Q_{k} \rightarrow M_{k-l}$ by

$$
\left\{\begin{array}{l}
\delta_{k, 1}=d \circ \epsilon_{k} \\
\delta_{k, 1}^{\prime}=d^{\prime} \circ \epsilon_{k}
\end{array}\right.
$$

and

$$
\left\{\begin{array}{l}
\delta_{k, l+1}=d \circ \delta_{k, l}^{\prime} \\
\delta_{k, l+1}^{\prime}=d^{\prime} \circ \delta_{k, l} .
\end{array}\right.
$$

Since each of its differentials is acyclic, the top row of the diagram below is an object of $B^{\mathrm{q}} \mathcal{P}$ for each $k \in\{0, \ldots, n\}$.

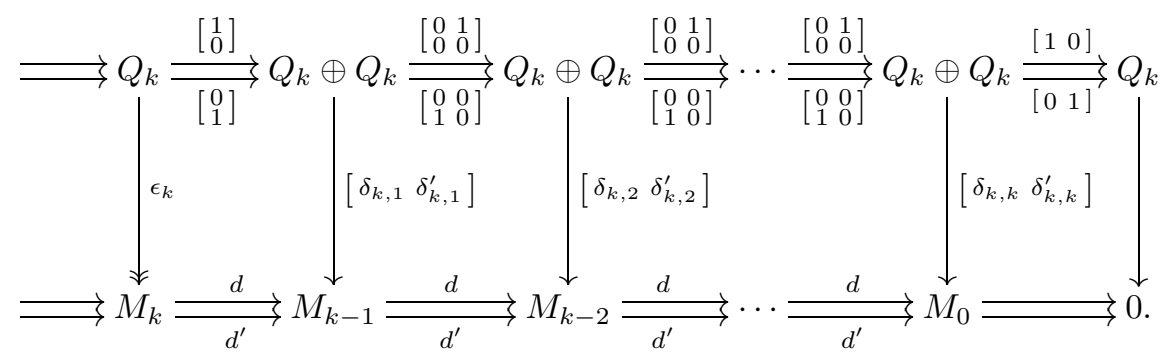

The morphisms $\delta_{k, l}$ and $\delta_{k, l}^{\prime}$ have been constructed so that the vertical morphisms commute with the top and bottom differentials, so the diagram represents a morphism in $B^{\mathrm{q}} \mathcal{M}$, which we shall again denote by $\zeta^{k}: P^{k} \rightarrow M$. Following the same method of proof as of the previous lemma, each $P^{k}$ is acyclic so their direct sum is acyclic as well and so

$$
\zeta:=\left[\begin{array}{lll}
\zeta^{n} & \cdots & \zeta^{0}
\end{array}\right]: \bigoplus P^{k} \rightarrow M
$$

is a morphism in $B^{\mathrm{q}} \mathcal{M}$. By construction and Lemma 3.3 again, each morphism $\zeta^{j}: P_{j} \rightarrow M_{j}$ is an admissible epimorphism in $\mathcal{M}$. Each of these morphisms therefore has a kernel in $\mathcal{P}$ and these kernels form a binary complex with the induced maps.

We wish to show that both differentials of this binary complex are acyclic in $\mathcal{P}$. Consider the top differential first. Denote the objects that the top differentials of $M, P$ and each $P^{k}$ factor through by $Z_{j}, Y_{j}$, and $Y_{j}^{k}$ (so $Y_{j}$ is the sum of all the factors $\left.Y_{j}^{k}\right)$. As in the proof of Lemma 3.5, it is enough to show that each induced morphism $Y_{j} \rightarrow Z_{j}$ is an admissible epimorphism of $\mathcal{M}$. But since $Y_{j} \rightarrow Z_{j}$ is equal to the product of induced maps $\bigoplus Y_{j}^{k} \rightarrow Z_{j}$, it suffices, by Lemma 3.3. to show that, for each $j$, one of the morphisms $Y_{j}^{k} \rightarrow Z_{j}$ is an admissible epimorphism of $\mathcal{M}$. Since 
$Y_{j}^{j}=Q_{j}$, taking $k=j$ yields the result, as shown by the diagram below.

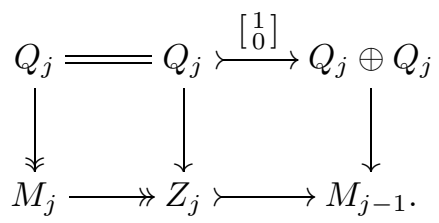

The bottom differential is dealt with entirely analogously.

Proof of Proposition 3.2. We proceed by induction on $n$. In the base case $n=0$, there is nothing to show. For the inductive step, we view an acyclic binary multicomplex $M$ in $\left(B^{\mathrm{q}}\right)^{n+1} \mathcal{M}$ as an acyclic binary multicomplex of objects of $\left(B^{\mathrm{q}}\right)^{n} \mathcal{M}$, i.e., as an object of $B^{q}\left(B^{\mathrm{q}}\right)^{n} \mathcal{M}$. By the inductive hypothesis, the inclusion of $\left(B^{\mathrm{q}}\right)^{n} \mathcal{P}$ into $\left(B^{\mathrm{q}}\right)^{n} \mathcal{M}$ satisfies the hypotheses of Theorem 3.1, so by Lemma 3.6 there exists a short exact sequence $0 \rightarrow P^{\prime} \rightarrow P \rightarrow M \rightarrow 0$ in $B^{q}\left(B^{\mathrm{q}}\right)^{n} \mathcal{M}$ with $P^{\prime}$ and $P$ in $B^{q}\left(B^{\mathrm{q}}\right)^{n} \mathcal{P}=$ $\left(B^{\mathrm{q}}\right)^{n+1} \mathcal{P}$, and so the first part follows. For the second part, suppose that $M$ is diagonal in some direction $i$. We consider $M$ as a diagonal acyclic binary complex of (not necessarily diagonal) objects of $\left(B^{\mathrm{q}}\right)^{n} \mathcal{M}$, that is, we "expand" $M$ along the $i$ direction. Then by Lemma 3.5 there exist diagonal acyclic binary complexes $P^{\prime}$ and $P$ in $\left(B^{\mathrm{q}}\right)^{n+1} \mathcal{P}$ that are diagonal in direction $i$, and an exact sequence $0 \rightarrow P^{\prime} \rightarrow$ $P \rightarrow M \rightarrow 0$, so the proof is complete.

\section{The cofinality theorem}

Unlike the additivity and resolution theorems, the cofinality theorem was not proved by Quillen in Qui73. A proof for exact categories based on work by Gersten was given in Gra79. More general versions can be found in Sta89 and TT90. It is proven in Gra12 that the hypotheses of the cofinality theorem are satisfied by the appropriate exact categories of acyclic binary complexes, the main work in our proof is in ensuring that the results pass to the quotients defining $K_{n}$.

Definition 4.1. An exact subcategory $\mathcal{M}$ of an exact category $\mathcal{N}$ is said to be cofinal in $\mathcal{N}$ if for every object $N_{1}$ of $\mathcal{N}$ there exists another object $N_{2}$ of $\mathcal{N}$ such that $N_{1} \oplus N_{2}$ is isomorphic to an object of $\mathcal{M}$.

An obvious example of a cofinal exact subcategory is the category of free $R$-modules inside the category of projective $R$-modules, for any ring $R$. More generally, every exact category is cofinal in its idempotent completion ([TT90], Appendix A). The cofinality theorem relates the $K$-theory of the cofinal subcategory to the $K$-theory of the exact category containing it. Throughout this section $\mathcal{M} \hookrightarrow \mathcal{N}$ is the inclusion of a cofinal exact subcategory of an exact category $\mathcal{N}$.

Define an equivalence relation on the objects of $\mathcal{N}$ by declaring $N_{1} \sim N_{2}$ if there exist objects $M_{1}$ and $M_{2}$ of $\mathcal{M}$ such that

$$
N_{1} \oplus M_{1} \cong N_{2} \oplus M_{2}
$$

Since $\langle M\rangle=0$ for every $M$ in $\mathcal{M}$, the cofinality of $\mathcal{M}$ in $\mathcal{N}$ ensures that equivalence classes of $\sim$ form a group under the natural operation $\left\langle N_{1}\right\rangle+\left\langle N_{2}\right\rangle=\left\langle N_{1} \oplus N_{2}\right\rangle$; we 
denote this group by $K_{0}(\mathcal{N}$ rel. $\mathcal{M})$. The following lemma and its corollary were first observed in the proof of Theorem 1.1 in Gra79.

Lemma 4.2. The sequence:

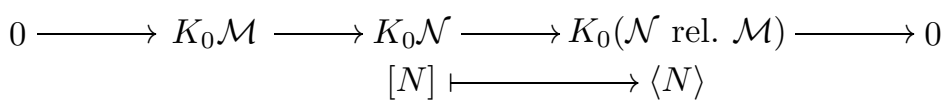

is well-defined and exact.

Corollary 4.3. For any pair of objects $N_{1}, N_{2}$ of $\mathcal{N}$ with the same class in $\operatorname{coker}\left(K_{0} \mathcal{M} \rightarrow K_{0} \mathcal{N}\right)$ there exists a (single) object $N^{\prime}$ in $\mathcal{N}$ such that each $N_{i} \oplus N^{\prime}$ is in $\mathcal{M}$.

Proof. By the lemma, if $N_{1}$ and $N_{2}$ have the same class in coker $\left(K_{0} \mathcal{M} \rightarrow K_{0} \mathcal{N}\right)$, then $\left\langle N_{1}\right\rangle=\left\langle N_{2}\right\rangle$. From cofinality there exists a $P$ in $\mathcal{N}$ such that $N_{1} \oplus P$ is in $\mathcal{M}$, so $\langle 0\rangle=\left\langle N_{1} \oplus P\right\rangle=\left\langle N_{2} \oplus P\right\rangle$. Hence there exist objects $P_{1}$ and $P_{2}$ of $\mathcal{M}$ such that each $\left(N_{i} \oplus P\right) \oplus P_{i}$ is an object of $\mathcal{M}$. Setting $N^{\prime}=P \oplus P_{1} \oplus P_{2}$, each $N_{i} \oplus N^{\prime}$ is an object of $\mathcal{M}$.

We show now that cofinality of $\mathcal{M}$ in $\mathcal{N}$ passes to the associated categories of acyclic binary multicomplexes.

Lemma 4.4. For every object $N$ of $B^{\mathrm{q}} \mathcal{N}$ there exists a diagonal object $T$ in $B^{\mathrm{q}} \mathcal{N}$ such that $N \oplus T$ is in $B^{\mathrm{q}} \mathcal{M}$.

Proof. This is Lemma 6.2 of Gra12.

Corollary 4.5. For each $n \geqslant 1$, the category $\left(B^{\mathrm{q}}\right)^{n} \mathcal{M}$ is cofinal in $\left(B^{\mathrm{q}}\right)^{n} \mathcal{N}$.

Proof. This follows by straightforward induction on the previous lemma (the hypothesis that $T$ is diagonal is not actually needed).

We can also conclude that $\left(C^{\mathrm{q}}\right)^{n} \mathcal{M}$ is cofinal in $\left(C^{\mathrm{q}}\right)^{n} \mathcal{N}$. This can be proved independently, but it also follows trivially from Corollary 4.5

These facts in hand, we now proceed to the proof of the cofinality theorem.

Theorem 4.6 (Cofinality). The inclusion functor $\mathcal{M} \hookrightarrow \mathcal{N}$ induces an injection $K_{0} \mathcal{M} \hookrightarrow K_{0} \mathcal{N}$ and isomorphisms $K_{n} \mathcal{M} \cong K_{n} \mathcal{N}$ for $n>0$.

Proof. The case $n=0$ is part of Lemma4.2 so we proceed directly to $n>0$. We consider $n=1$ first. The inclusion $\mathcal{M} \hookrightarrow \mathcal{N}$ induces a morphism of short exact sequences:

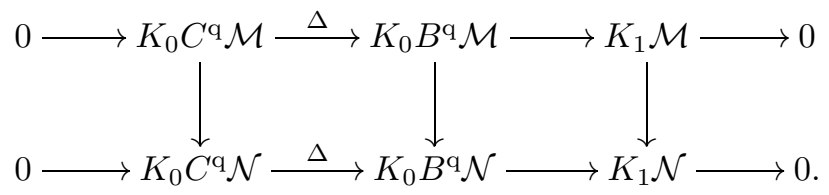

By Lemma 2.7 these short exact sequences are split. Furthermore, the downwards maps commute with the splittings of $\Delta\left(\top: K_{0} B^{\mathrm{q}} \mathcal{M} \rightarrow K_{0} C^{\mathrm{q}} \mathcal{M}\right.$ and $\mathrm{\top}: K_{0} B^{\mathrm{q}} \mathcal{N} \rightarrow$ $\left.K_{0} C^{\mathrm{q}} \mathcal{N}\right)$, so the morphism is a morphism of split exact sequences. The induced map 
$K_{0} B^{\mathrm{q}} \mathcal{M} \rightarrow K_{0} B^{\mathrm{q}} \mathcal{N}$ is a monomorphism, as $K_{0} B^{\mathrm{q}} \mathcal{M}$ is cofinal in $K_{0} B^{\mathrm{q}} \mathcal{N}$, by Corollary 4.5. The map $K_{1} \mathcal{M} \rightarrow K_{1} \mathcal{N}$ is therefore a direct summand of a monomorphism and must be a monomorphism itself.

The induced map $K_{1} \mathcal{M} \rightarrow K_{1} \mathcal{N}$ sends the class $x+K_{0} C^{\mathrm{q}} \mathcal{M} \in K_{1} \mathcal{M}$ to the class $x+K_{0} C^{\mathrm{q}} \mathcal{N} \in K_{1} \mathcal{N}$, where $x \in K_{0} B^{\mathrm{q}} \mathcal{M}$. Let $x=\left[N_{1}\right]-\left[N_{2}\right]$ be a generic element of $K_{0} B^{\mathrm{q}} \mathcal{N}$, where $N_{1}$ and $N_{2}$ are acyclic binary complexes with objects in $\mathcal{N}$. By Lemma 4.4, there exist diagonal acyclic binary complexes $T_{1}$ and $T_{2}$ in $B^{\mathrm{q}} \mathcal{N}$ such that $N_{i} \oplus T_{i} \in B^{\mathrm{q}} \mathcal{M}$ for $i=1,2$. Then

$$
\left[N_{i}\right]+K_{0} C^{\mathrm{q}} \mathcal{N}=\left[N_{i} \oplus T_{i}\right]+K_{0} C^{\mathrm{q}} \mathcal{N}
$$

and therefore

$$
x+K_{0} C^{\mathrm{q}} \mathcal{N}=\left[N_{1}\right]-\left[N_{2}\right]+K_{0} C^{\mathrm{q}} \mathcal{N}=\left[N_{1} \oplus T_{1}\right]-\left[N_{2} \oplus T_{2}\right]+K_{0} C^{\mathrm{q}} \mathcal{N}
$$

is in the image of $K_{1} \mathcal{M} \rightarrow K_{1} \mathcal{N}$. Thus $K_{1} \mathcal{M} \rightarrow K_{1} \mathcal{N}$ is surjective and the case $n=1$ of the theorem is proven.

The remaining cases now follow by an induction on $n$. By Lemma 2.7 again, the inclusion $\mathcal{M} \hookrightarrow \mathcal{N}$ induces a morphism of split short exact sequences:

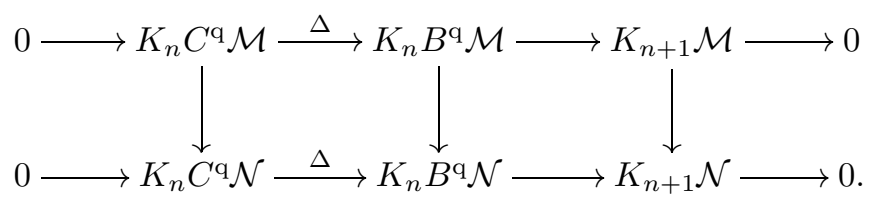

If $n \geqslant 1$, the induced map $K_{n} B^{\mathrm{q}} \mathcal{M} \rightarrow K_{n} B^{\mathrm{q}} \mathcal{N}$ is an isomorphism by the inductive hypothesis, since $B^{\mathrm{q}} \mathcal{M}$ is cofinal in $K_{n} B^{\mathrm{q}} \mathcal{N}$, by Lemma 4.4 again. Therefore $K_{n+1} \mathcal{M} \rightarrow K_{n+1} \mathcal{N}$ is a direct summand of an isomorphism, so it is itself an isomorphism.

Remark 4.7. It is possible to give a proof of the cofinality theorem that does not use Lemma 2.7, although it is less natural than the above. The intermediate steps may be of independent interest, so we outline this alternative argument below. Details will be included in the author's $\mathrm{PhD}$ thesis Har15.

Alternative proof of cofinality theorem (outline). By modifying the proof of Lemma 6.2 in Gra12, one can prove the following (stronger) variant of our Lemma 4.4.

For all $n \geqslant 1$, if $N$ is in $\left(B^{\mathrm{q}}\right)^{n} \mathcal{N}$ and $i \in\{1, \ldots, n\}$ is any direction, then there exists an object $T$ in $\left(B^{\mathrm{q}}\right)^{n} \mathcal{N}$ that is diagonal in direction $i$ such that $N \oplus T$ is in $\left(B^{\mathrm{q}}\right)^{n} \mathcal{M}$. Moreover, if $N$ is diagonal in direction $j \in\{1, \ldots, n\}, j \neq i$, then $T$ may be chosen to be diagonal in both direction $i$ and direction $j$.

This can then be used to prove the following statement:

Let $x+T_{\mathcal{M}}^{n}$ be a class in $T_{\mathcal{N}}^{n} / T_{\mathcal{M}}^{n}$, for $n \geqslant 1$. Then $x+T_{\mathcal{M}}^{n}=[t]+T_{\mathcal{M}}^{n}$, where $[t]$ is the class in $K_{0}\left(B^{\mathrm{q}}\right)^{n} \mathcal{N}$ of a single diagonal acyclic binary multicomplext in $\left(B^{\mathrm{q}}\right)^{n} \mathcal{N}$.

From this one can deduce that the canonical map

$$
T_{\mathcal{N}}^{n} / T_{\mathcal{M}}^{n} \longrightarrow K_{0}\left(B^{\mathrm{q}}\right)^{n} \mathcal{N} / K_{0}\left(B^{\mathrm{q}}\right)^{n} \mathcal{M}
$$

is injective. The surjectivity of this map is an evident application of the abovementioned variant of Lemma 4.4. Finally, a straightforward argument using the snake 
lemma shows that induced homomorphism $K_{n} \mathcal{M} \rightarrow K_{n} \mathcal{N}$ is bijective for $n \geqslant 1$.

\section{References}

[Büh10] T. Bühler, Exact categories, Expo. Math. 28 (2010), no. 1, 1-69.

[Gra79] D.R. Grayson, Localization for flat modules in algebraic $K$-theory, $J$. Algebra 61 (1979), no. 2, 463-496.

[Gra11] , The additivity theorem in algebraic K-theory, Doc. Math. 16 (2011), 457-464.

[Gra12] Algebraic $K$-theory via binary complexes, J. Amer. Math. Soc. 25 (2012), no. 4, 1149-1167.

[Gra13] Relative algebraic $K$-theory by elementary means, pre-print (2013), http://arxiv.org/abs/1310.8644

[Har15] T. Harris, Binary complexes and algebraic $K$-theory, $\mathrm{PhD}$ thesis, in preparation.

[McC93] R. McCarthy, On fundamental theorems of algebraic $K$-theory, Topology 32 (1993), no. 2, 325-328.

[Qui73] D. Quillen, Higher algebraic K-theory. I, Algebraic K-theory, I: Higher K-theories (Proc. Conf., Battelle Memorial Inst., Seattle, Wash., 1972), Springer, Berlin, 1973, pp. 85-147. Lecture Notes in Math., Vol. 341.

[Sta89] R.E. Staffeldt, On fundamental theorems of algebraic $K$-theory, $K$ Theory 2 (1989), no. 4, 511-532.

[TT90] R.W. Thomason and Thomas Trobaugh, Higher algebraic $K$-theory of schemes and of derived categories, The Grothendieck Festschrift, Vol. III, Progr. Math., vol. 88, Birkhäuser Boston, Boston, MA, 1990, pp. 247-435.

[Wei13] C.A. Weibel, The $K$-book: an introduction to algebraic $K$-theory, Graduate Studies in Mathematics, vol. 145, Amer Mathematical Society, Providence, R.I., 2013.

Tom Harris tkmharris@gmail.com

Mathematical Sciences, University of Southampton, Southampton SO17 1BJ, UK 